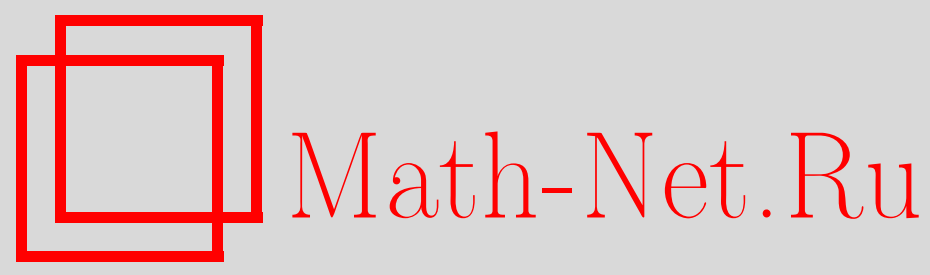

С. П. Хэкало, Пошаговая калибровочная эквивалентность дифференциальных операторов, Матем. заметки, 2005, том 77, выпуск 6, 917-929

DOI: https://doi.org/10.4213/mzm2541

Использование Общероссийского математического портала Math-Net.Ru подразумевает, что вы прочитали и согласны с пользовательским соглашением http://www . mathnet.ru/rus/agreement

Параметры загрузки:

IP: 3.85 .5 .30

26 апреля 2023 г., $17: 21: 08$

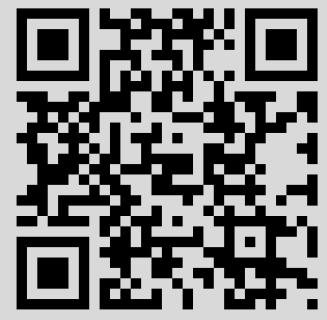




\section{ПОШАГОВАЯ КАЛИБРОВОЧНАЯ ЭКВИВАЛЕНТНОСТЬ ДИФФЕРЕНЦИАЛЬНЫХ ОПЕРАТОРОВ}

\section{С. П. Хэкало}

В работе изучается зависимость между понятием калибровочной эквивалентности и решениями некоторых систем нелинейных дифференциальных уравнений в частных производных. Эта связь основана на пошаговой калибровочной эквивалентности.

Библиография: 16 названий.

1. Введение. Одним из методов построения дифференциальных операторов, удовлетворяющих принципу Гюйгенса [1], [2], явились деформации априори гюйгенсовых операторов. Первые шаги в этом направлении фактически осуществили Штелльмахер и Лагнезе [3], [4].

В работах [1], [5]-[7] было отмечено, что известные примеры деформаций гюйгенсовых операторов подчиняются так назьваемому свойству калибровочной эквивалентности дифференциальных операторов [1], [7]. Это свойство является своеобразным “конкурентом" критерию Адамара для проверки оператора на вьполнение принципа Гюйгенса (критерий Адамара состоит в том, что начиная с некоторого номера, соответствующий оператору анзатц имеет нулевые коэффициенты).

В отличие от критерия А дамара свойство калибровочной эквивалентности обладает следуюшим преимуществом: применение анзатца Адамара возможно лишь к гиперболическим дифференциальным операторам второго порядка и сравнительно небольшому классу других операторов [8], а на калибровочную эквивалентность можно исследовать произвольные дифференциальные операторы. Заметим, однако, что проверка калибровочной эквивалентности операторов затруднительна, и ее наличие дает лиш достаточное условие выполнения принципа Гюйгенса.

В работе [9] на основе теории биспектральных операторов, решена задача о калибровочной эквивалентности обыкновенных дифференциальных операторов.

В настоящей заметке предлагается способ пошаговой калибровочной эквивалентности, применяемый к линейньп дифференциальным операторам в частных производных, зависящим от неотрицательных целых параметров.

Фактически, этот способ позволяет свести вопрос о калибровочной эквивалентности дифференциальных операторов к решению системы нелинейных дифференциальных уравнений в частных производных. При решении такой системы в явном виде получаются коэффициенты изодеформаций априори гюйгенсовых операторов.

Работа выполнена при поддержке Российского фонда фундаментальных исследований, грант № 05-01-00248, и Фонда содействия отечественной науке. 
2. Определение и свойства калибровочной эквивалентности. Пусть $\mathscr{A}, \mathscr{B}$ и $\mathscr{C}$ - произвольные операторы на $\mathbb{R}^{N}, N \geqslant 1$, 'о' - знак композиции операторов.

Оператором косого присоединенного действия [10], [11] назьвается оператор $\operatorname{ad}_{\mathscr{A}, \mathscr{B}}^{k} \mathscr{C}, k \in \mathbb{Z}_{+}$, определяемьй следуюшим рекуррентньм соотношением:

$$
\operatorname{ad}_{\mathscr{A}, \mathscr{B}}^{0} \mathscr{C}=\mathscr{C}, \quad \operatorname{ad}_{\mathscr{A}, \mathscr{B}}^{k} \mathscr{C}=\operatorname{ad}_{\mathscr{A}, \mathscr{B}}^{k-1}(\mathscr{A} \circ \mathscr{C}-\mathscr{C} \circ \mathscr{B}), \quad k \in \mathbb{N}
$$

ОПРЕДЕЛЕниЕ [10]. Дифференциальньй оператор $\mathscr{L}_{2}$ назьвается $M$-калибровочно эквивалентным оператору $\mathscr{L}_{1}$ на $\mathbb{R}^{N}$ (при помощи функции $f$ ), если найдутся натуральное число $M$ и ненулевая бесконечно дифференцируемая функция $f$ такие, что

$$
\operatorname{ad}_{\mathscr{L}_{2}, \mathscr{L}_{1}}^{M+1} f=0
$$

Пусть $\mathscr{L}_{1}, \mathscr{L}_{2}$ - линейные дифференциальные операторы на $\mathbb{R}^{N}$ и

$$
\mathscr{L}_{2}=\sum_{\alpha,|\alpha| \leqslant K_{2}} u_{\alpha}(x) \partial^{\alpha},
$$

где $\alpha=\left(\alpha_{1}, \ldots, \alpha_{N}\right)$ - мультииндекс целых неотрицательных чисел; $|\alpha|=\alpha_{1}+\cdots+\alpha_{N}$, $K_{i}$-степень оператора $\mathscr{L}_{i}, u(x)=\left\{u_{\alpha}(x)\right\}$-некоторые кусочно-гладкие коэффициенты оператора $\mathscr{L}_{2} ; \partial^{\alpha}=\partial^{|\alpha|} / \partial x_{1}^{\alpha} \cdots \partial x_{N}^{\alpha}$. Тогда оператор $\operatorname{ad}_{\mathscr{L}_{2}, \mathscr{L}_{1}}^{M+1} f$ можно записать в неявной форме

$$
\operatorname{ad}_{\mathscr{L}_{2}, \mathscr{L}_{1}}^{M+1} f=\sum_{\alpha,|\alpha| \leqslant K} U_{\alpha, \mathscr{L}_{1}}\left(\partial^{\beta} u(x), \partial^{\gamma} f, M\right) \partial^{\alpha},
$$

где $\partial^{\beta} u(x)$ - частные производные порядка $|\beta| \leqslant M \cdot K_{2}$ коэффициентов оператора $\mathscr{L}_{2}$, $\partial^{\gamma} f$ - частные производные порядка $|\gamma| \leqslant M \cdot K_{2}$ функции $f$.

Таким образом, в случае, когда по заданному оператору $\mathscr{L}_{1}$ надо построить ему калибровочно эквивалентньй оператор $\mathscr{L}_{2}$, нужно решить систему нелинейных дифференциальных уравнений в частных производных

$$
U_{\alpha, \mathscr{L}_{1}}\left(\partial^{\beta} u(x), \partial^{\gamma} f, M\right)=0, \quad|\alpha| \leqslant K \leqslant\left(\max \left\{K_{1}, K_{2}\right\}\right)^{M+1},
$$

на коэффициенты оператора $\mathscr{L}_{2}$, функцию $f$ и параметр $M$. В настоящей статье это обстоятельство будет играть главную роль в построении калибровочно эквивалентных операторов.

Сформулируем основные утверждения о калибровочной эквивалентности операторов. Пусть $\kappa(\mathscr{L})$ - главньй символ оператора $\mathscr{L}$.

УТВЕРЖДЕНИЕ 1. Пусть $\mathscr{L}_{1}$ и $\mathscr{L}_{2}$ - линейные дифференциальные операторы. Тогда для любого $m \in \mathbb{N}$ имеет место равенство

$$
\kappa\left(\operatorname{ad}_{\mathscr{L}_{2}, \mathscr{L}_{1}}^{m} f\right)=f\left(\kappa\left(\mathscr{L}_{2}\right)-\kappa\left(\mathscr{L}_{1}\right)\right)^{m} .
$$

Действительно, оператор косого присоединенного действия можно записать в следующей форме:

$$
\operatorname{ad}_{\mathscr{L}_{2}, \mathscr{L}_{1}}^{m} f=\sum_{k=0}^{m}(-1)^{k}\left(\begin{array}{c}
m \\
k
\end{array}\right) \mathscr{L}_{2}^{m-k} \circ f \circ \mathscr{L}_{1}^{k} .
$$


Тогда

$$
\begin{aligned}
\kappa\left(\operatorname{ad}_{\mathscr{L}_{2}, \mathscr{L}_{1}}^{m} f\right) & =\sum_{k=0}^{m}(-1)^{k}\left(\begin{array}{c}
m \\
k
\end{array}\right) \kappa\left(\mathscr{L}_{2}^{m-k}\right) \kappa(f) \kappa\left(\mathscr{L}_{1}^{k}\right) \\
& =\kappa(f) \sum_{k=0}^{m}(-1)^{k}\left(\begin{array}{c}
m \\
k
\end{array}\right) \kappa\left(\mathscr{L}_{2}\right)^{m-k} \kappa\left(\mathscr{L}_{1}\right)^{k}=f\left(\kappa\left(\mathscr{L}_{2}\right)-\kappa\left(\mathscr{L}_{1}\right)\right)^{m} .
\end{aligned}
$$

Следующее утверж дение показьвает, что калибровочно эквивалентными если и могут быть, то лишш оператор и его деформация членами меньшего порядка.

УТВЕРЖДЕНИЕ 2. Если линейные операторы $\mathscr{L}_{2}$ и $\mathscr{L}_{1}$ калибровочно эквивалентны, то $\kappa\left(\mathscr{L}_{2}\right)=\kappa\left(\mathscr{L}_{1}\right)$.

Утверждение 2 следует из утверждения 1 . Оно также очевидно следует и из определения калибровочной эквивалентности:

$$
\operatorname{ad}_{\mathscr{L}_{2}, \mathscr{L}_{1}}^{M+1} f=\mathscr{L}_{2} \circ\left(\operatorname{ad}_{\mathscr{L}_{2}, \mathscr{L}_{1}}^{M} f\right)-\left(\operatorname{ad}_{\mathscr{L}_{2}, \mathscr{L}_{1}}^{M} f\right) \circ \mathscr{L}_{1}=0
$$

УТВЕРЖДЕНИЕ 3 [5]. Оператор $\mathscr{L}_{2} \quad M$-калибровочно әквивалентен оператору $\mathscr{L}_{1}$ (при помощи функиии $f$ ) тогда и только тогда, когда найдется ненулевая бесконечно дифференцируемая функиия $f$ такая, что дифференциальный оператор

$$
\mathscr{D}_{t}^{M}(f)=\sum_{k=0}^{M} \frac{1}{k !}\left(\operatorname{ad}_{\left.\mathscr{L}_{2}, \mathscr{L}_{1} f\right) t^{k}}^{k}\right.
$$

со спектральным параметром $t \in \mathbb{R}$ будет сплетающим для операторов $\mathscr{L}_{2}$ и $\mathscr{L}_{1}$ на $\mathbb{R}^{N} \times \mathbb{R}:$

$$
\left(\frac{\partial}{\partial t}-\mathscr{L}_{2}\right) \circ \mathscr{D}_{t}^{M}(f)=\mathscr{D}_{t}^{M}(f) \circ\left(\frac{\partial}{\partial t}-\mathscr{L}_{1}\right) .
$$

ЗАмЕчАнИЕ. Пусть $\mathscr{L}_{1}$ - линейный дифференциальньй оператор с постоянными коэффициентами на $\mathbb{R}^{N}$, и пусть $\xi$ - фиксированная точка $\mathbb{R}^{N},\langle\xi, \cdot\rangle$ - стандартное скалярное произведение на $\mathbb{R}^{N}$. Тогда из того, что оператор $\mathscr{L}_{2} M$-калибровочно эквивалентен оператору $\mathscr{L}_{1}$ с помощью некоторой фиксированной функции $f$ следует, что функция

$$
\psi_{\xi, f}(x)=\operatorname{ad}_{\mathscr{L}_{2}, \mathscr{L}_{1}}^{M} f\left[e^{\langle\xi, x\rangle}\right]
$$

является собственной функцией оператора $\mathscr{L}_{2}$. Собственное значение при этом есть значение символа $\widetilde{\kappa}\left(\mathscr{L}_{1}\right)$ оператора $\mathscr{L}_{1}$ в точке $\xi$. В самом деле,

$$
\begin{aligned}
\mathscr{L}_{2}\left[\psi_{\xi, f}(x)\right] & =\mathscr{L}_{2}\left[\operatorname{ad}_{\mathscr{L}_{2}, \mathscr{L}_{1}}^{M} f\left[e^{\langle\xi, x\rangle}\right]\right]=\left(\mathscr{L}_{2} \circ \operatorname{ad}_{\left.\mathscr{L}_{2}, \mathscr{L}_{1} f\right)\left[e^{\langle\xi, x\rangle}\right]}^{M}\right. \\
& \left.=\operatorname{ad}_{\mathscr{L}_{2}, \mathscr{L}_{1}}^{M} f \circ \mathscr{L}_{1}\right)\left[e^{\langle\xi, x\rangle}\right]=\operatorname{ad}_{\mathscr{L}_{2}, \mathscr{L}_{1}}^{M} f\left[\mathscr{L}_{1}\left[e^{\langle\xi, x\rangle}\right]\right] \\
& =\operatorname{ad}_{\mathscr{L}_{2}, \mathscr{L}_{1}}^{M} f\left[\widetilde{\kappa}\left(\mathscr{L}_{1}\right)(\xi) \cdot e^{\langle\xi, x\rangle}\right]=\widetilde{\kappa}\left(\mathscr{L}_{1}\right)(\xi) \cdot \psi_{\xi, f}(x) .
\end{aligned}
$$

Далее, для оператора $\mathscr{L}_{1}$ на $\mathbb{R}^{N}$ пусть $\left\{\Phi_{1}^{(\lambda)}(x), x \in \mathbb{R}^{N}\right\}_{\lambda \in \mathbb{C}}-$ семейство целых по $\lambda$ обобщенных функций, задающее ядро Рисса:

$$
\mathscr{L}_{1} \Phi_{1}^{(\lambda)}(x)=\Phi_{1}^{(\lambda-1)}(x), \quad \Phi_{1}^{(0)}(x)=\delta(x),
$$


где $\delta$ - дельта-функция Дирака. Это значит, что для операторов $\mathscr{L}_{1}^{k}, k \in \mathbb{N}$, известны фундаментальные решения

$$
\mathscr{L}_{1}^{k} \Phi_{1}^{(k)}(x-\xi)=\delta(x-\xi),
$$

где $\xi \in \mathbb{R}^{N}$ - фиксированная точка.

Важность понятия калибровочной эквивалентности заключается в следующем: построение фундаментального решения деформированного оператора в случае его калибровочной эквивалентности с исходньм можно осуществлять на основе фундаментальных решений степеней исходного оператора (это построение возможно в явном виде).

Следующее утверждение фактически получено в [2].

УТВЕРЖДЕНИЕ 4. Пусть оператор $\mathscr{L}_{2}$ М-калибровочно әквивалентен оператору $\mathscr{L}_{1}$. Тогда семейство

$$
\left\{\left.\mathscr{H}_{\partial_{t}}^{M}(f) \Phi_{2}^{(\lambda)}(x, \xi ; t)\right|_{t=0}, x, \xi \in \mathbb{R}^{N} ; t \in \mathbb{R}\right\}_{\lambda \in \mathbb{C}}
$$

$2 \partial e$

$$
\begin{aligned}
\mathscr{H}_{\partial_{t}}^{M}(f) & =\sum_{k=0}^{M} \frac{(-1)^{k}}{k !}\left(\operatorname{ad}_{\mathscr{L}_{2}, \mathscr{L}_{1}}^{k} f\right) \frac{\partial^{k}}{\partial t^{k}}, \\
\Phi_{2}^{(\lambda)}(x, \xi ; t) & =\frac{1}{f(\xi)} \sum_{i=0}^{Q}\left(\begin{array}{c}
\lambda+i-1 \\
i
\end{array}\right) \Phi_{1}^{(\lambda+i)}(x-\xi) t^{i}
\end{aligned}
$$

$\left(Q\right.$ достаточно велико) задает ядро Рисса для оператора $\mathscr{L}_{2}$ :

$$
\begin{aligned}
\mathscr{L}_{2}\left(\left.\mathscr{H}_{\partial_{t}}^{M}(f) \Phi_{2}^{(\lambda)}(x, \xi ; t)\right|_{t=0}\right) & =\left.\mathscr{H}_{\partial_{t}}^{M}(f) \Phi_{2}^{(\lambda-1)}(x, \xi ; t)\right|_{t=0}, \\
\left.\mathscr{H}_{\partial_{t}}^{M}(f) \Phi_{2}^{(0)}(x, \xi ; t)\right|_{t=0} & =\delta(x-\xi) .
\end{aligned}
$$

СлЕдСтвиЕ. Onератор $\mathscr{L}_{2}^{k}, k \in \mathbb{N}$, имеет фундаментальным решением обобщенную функиию $\left.\mathscr{H}_{\partial_{t}}^{M}(f) \Phi_{2}^{(k)}(x, \xi ; t)\right|_{t=0}$ :

$$
\mathscr{L}_{2}^{k}\left(\left.\mathscr{H}_{\partial_{t}}^{M}(f) \Phi_{2}^{(k)}(x, \xi ; t)\right|_{t=0}\right)=\delta(x-\xi) .
$$

ЗАмечАниЕ. В случае, когда оператор $\mathscr{L}_{1}$ имеет ядро Рисса, необходимое и достаточное условие калибровочной эквивалентности операторов $\mathscr{L}_{2}$ и $\mathscr{L}_{1}$ состоит в существовании у оператора $\mathscr{L}_{2}$ “пси-функции" $\psi_{\xi}(x)=F_{\xi}(x) e^{\langle\xi, x\rangle}$ с рациональным ядром $F_{\xi}(x)$.

3. Основной результат. Если оператор $\mathscr{L}_{1}$ и некоторые его степени удовлетворяют принципу Гюйгенса, то утверждение 3 дает достаточные условия выполнения принципа Гюйгенса деформированного оператора $\mathscr{L}_{2}$. Таким образом, для конструирования новых операторов, удовлетворяющих принципу Гюйгенса, достаточно решить вопрос об эффективном построении калибровочно эквивалентных операторов.

В работах [1], [2], [5] этот вопрос решается путем привлечения теории операторов Данкла, связанных с корневыми системами в $\mathbb{R}^{N}$ и дальнейшей проверкой критериев калибровочной эквивалентности. На этой основе в указанных работах получены значительные результаты в области изогюйгенсовьх деформаций.

В следующей теореме, анонсированной в [12], [13], описьваются условия пошаговой калибровочной эквивалентности операторов, зависящих от неотрицательных целых параметров. 
ТЕОремА 1 (достаточное условие калибровочной эквивалентности). Пусть $\mathscr{R}_{n}-$ линейный дифференциальный оператор на $\mathbb{R}^{N}$, зависящий от неотрицательного челого параметра п. Если для любого $k=1,2, \ldots, n$ оператор $\mathscr{R}_{k}$ 1-калибровочно әквивалентен оператору $\mathscr{R}_{k-1}$ при помощи функиии $f$, m.е.

$$
\operatorname{ad}_{\mathscr{R}_{k}, \mathscr{R}_{k-1}}^{1+1} f=0,
$$

то оператор $\mathscr{R}_{n} \quad n$-калибровочно әквивалентен оператору $\mathscr{R}_{0}$ при помощи функu,uu $f^{n}$

$$
\operatorname{ad}_{\mathscr{R}_{n}, \mathscr{R}_{0}}^{n+1} f=0 .
$$

ДокАЗАТЕЛЬСТво. Пусть для $k=1,2, \ldots, n$ выполняется равенство

$$
\operatorname{ad}_{\mathscr{R}_{k}, \mathscr{R}_{k-1}}^{1+1} f=0 .
$$

Тогда для спектрального параметра $t \in \mathbb{R}$ согласно утверждению 3 имеем на $\mathbb{R}^{N} \times \mathbb{R}$ равенство

$$
\left(\frac{\partial}{\partial t}-\mathscr{R}_{k}\right) \circ\left(f+t\left(\operatorname{ad}_{\mathscr{R}_{k}}, \mathscr{R}_{k-1} f\right)\right)=\left(f+t\left(\operatorname{ad}_{\mathscr{R}_{k}, \mathscr{R}_{k-1}} f\right)\right) \circ\left(\frac{\partial}{\partial t}-\mathscr{R}_{k-1}\right) .
$$

Таким образом,

$$
\left(\frac{\partial}{\partial t}-\mathscr{R}_{n}\right) \circ \mathscr{T}_{t}^{n}(f)=\mathscr{T}_{t}^{n}(f) \circ\left(\frac{\partial}{\partial t}-\mathscr{R}_{0}\right),
$$

где сплетающий оператор $\mathscr{T}_{t}^{n}(f)$ задается равенством

$$
\mathscr{T}_{t}^{n}(f)=\prod_{k=1}^{n}\left(f+t\left(\operatorname{ad} \mathscr{R}_{n-k+1}, \mathscr{R}_{n-k} f\right)\right)
$$

со свободным от $t$ оператором $\mathscr{T}_{0}^{n}(f)=f^{n}$.

Выполнение последнего сплетающего соотношения по утверждению 3 влечет вьполнение равенства

$$
\operatorname{ad}_{\mathscr{R}_{n}, \mathscr{R}_{0}}^{n+1} \mathscr{T}_{0}^{n}(f)=0
$$

что и утверждалось.

Теорема 1 может быть естественным образом обобщена на случай нескольких целых неотрицательных параметров.

Пусть $\overline{1}_{i}-$ мультииндекс, состоящий из нулевых элементов, за исключением единицы на $i$-й позиции.

ТЕОРемА 1'. Пусть $\bar{n}$ - мультииндекс фиксированных неотрииательных иелых параметров, $\bar{n}=\left(n_{1}, \ldots, n_{s}\right), s=1,2, \ldots, \mathscr{R}_{\bar{n}}-$ линейный дифференциальный оператор на $\mathbb{R}^{N}$, зависящий от $\bar{n}$. Если для любих $k_{i}=1,2, \ldots, n_{i}, \quad i=1, \ldots, s$, оператор $\mathscr{R}_{\bar{k}}$ 1-калибровочно әквивалентен оператору $\mathscr{R}_{\bar{k}_{-}-\overline{1}_{i}}$ при помощи функu,uu $f_{i}$, mo

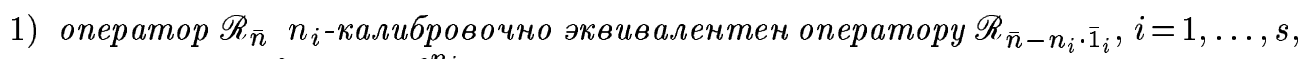
при помощи функиии $f_{i}^{n_{i}}$

2) оператор $\mathscr{R}_{\bar{n}}|\bar{n}|$-калибровочно әквивалентен оператору $\mathscr{R}_{\overline{0}}$ при помощи функиии $f^{\bar{n}}$. 
ДокАЗАТЕЛЬСТво. Если для любых $k_{i}=1,2, \ldots, n_{i}, i=1, \ldots, s$, оператор $\mathscr{R}_{\bar{k}}$ 1 -калибровочно эквивалентен оператору $\mathscr{R}_{\bar{k}-\overline{1}_{i}}$ при помощи функции $f_{i}$, то

$$
\operatorname{ad}_{\mathscr{R}_{\bar{k}}, \mathscr{R}_{\bar{k}-\overline{1}_{i}}^{1+1}}^{1+} f_{i}=0
$$

Тогда по теореме 1 для любых $i=1, \ldots, s$ имеем

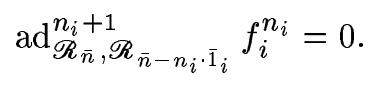

Первая часть теоремы $1^{\prime}$ доказана.

Вторая часть получается применением теоремы 1 для с двигов индекса оператора $\mathscr{R}_{\bar{n}}$, например, в следующем порядке:

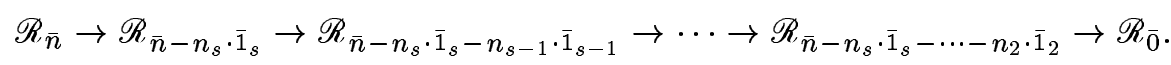

В результате

$$
\operatorname{ad}_{\mathscr{R}_{\bar{n}}, \mathscr{R}_{\overline{0}}}^{|\bar{n}|+1} f^{\bar{n}}=0, \quad|\bar{n}|=n_{1}+\cdots+n_{s}, \quad f^{\bar{n}}=\prod_{i=1}^{s} f_{i}^{n_{i}}
$$

и доказательство завершено.

Условия теоремы 1, как уже отмечалось вьше, равносильны системе нелинейных дифференциальных уравнений в частных производных с неотрицательными целыми параметрами. Однако в отличие от $M$-калибровочной эквивалентности, условие 1-калибровочной эквивалентности может быть вьписано в явном виде.

В самом деле, пусть $\mathscr{R}_{0}$ - однородный линейньй дифференциальный оператор порядка $K$ на $\mathbb{R}^{N}$ с постоянньми коэффициентами

$$
\mathscr{R}_{0}=\sum_{\alpha,|\alpha|=K} a_{\alpha} \partial^{\alpha}
$$

Здесь, как и ранее, $\alpha=\left(\alpha_{1}, \ldots, \alpha_{N}\right)$ - мультииндекс целых неотрицательных чисел; $|\alpha|=\alpha_{1}+\cdots+\alpha_{N}, \partial^{\alpha}=\partial^{|\alpha|} / \partial x_{1}^{\alpha_{1}} \cdots \partial x_{N}^{\alpha_{N}}$.

На основе необходимого условия калибровочной эквивалентности определим деформацию оператора $\mathscr{R}_{0}$, зависящую от неотрицательного целого параметра $n$ :

$$
\mathscr{R}_{n}=\sum_{\alpha,|\alpha| \leqslant K} u_{\alpha}^{n}(x) \partial^{\alpha}
$$

где на почти всюду гладкие коэффициенты $u_{\alpha}^{n}(x)$ накладываются ограничения

$$
\begin{gathered}
u_{\alpha}^{0}(x)= \begin{cases}a_{\alpha}, & |\alpha|=K, \\
0, & |\alpha|<K,\end{cases} \\
u_{\alpha}^{n}(x)=u_{\alpha}^{0}(x)=a_{\alpha}, \quad|\alpha|=K, \quad n=1,2, \ldots .
\end{gathered}
$$


Для $f \in C^{\infty}\left(\mathbb{R}^{N}\right), f \neq 0$, положим

$$
g_{\alpha}^{n}(f) \equiv g_{\alpha}^{n}=\sum_{\beta \geqslant \alpha,|\beta| \leqslant K}\left(\begin{array}{l}
\beta \\
\alpha
\end{array}\right) u_{\beta}^{n}\left(\partial^{\beta-\alpha} f\right)-u_{\alpha}^{n-1} f, \quad|\alpha| \leqslant K
$$

где биномиальный коэффициент на мультииндексах

$$
\left(\begin{array}{l}
\beta \\
\alpha
\end{array}\right)=\left(\begin{array}{l}
\beta_{1} \\
\alpha_{1}
\end{array}\right) \cdots\left(\begin{array}{l}
\beta_{N} \\
\alpha_{N}
\end{array}\right)
$$

задается произведением биномиальных коэффициентов и частичное упорядочение задается правилом

$$
\beta \geqslant \alpha \Longleftrightarrow \beta_{i} \geqslant \alpha_{i}, \quad i=1, \ldots, N \text {. }
$$

Далее, для мультииндексов $\beta$ и $\alpha$ таких, что $|\beta| \leqslant K,|\alpha| \leqslant K$, положим

$$
r_{\alpha \beta}^{n}(f) \equiv r_{\alpha \beta}^{n}=\sum_{\gamma \geqslant \alpha,|\gamma| \leqslant K}\left(\begin{array}{l}
\gamma \\
\alpha
\end{array}\right)\left[u_{\gamma}^{n}\left(\partial^{\gamma-\alpha} g_{\beta}^{n}\right)-g_{\gamma}^{n}\left(\partial^{\gamma-\alpha} u_{\beta}^{n-1}\right)\right]
$$

Выпишем некоторые свойства элементов $r_{\alpha \beta}$.

Свойство 1. Если $|\alpha|=|\beta|=K$, mo

$$
r_{\alpha \beta}=0
$$

Свойство 2. Если $|\alpha|=K$, mo

$$
r_{\alpha \beta}=a_{\alpha} g_{\beta}^{n}, \quad r_{\alpha \alpha}=0, \quad r_{\beta \alpha}=-a_{\alpha} g_{\beta}^{n}, \quad r_{\alpha \beta}+r_{\beta \alpha}=0
$$

ДоКАЗАТЕЛЬСТВО этих утверждений непосредственно следует из определения элементов $r_{\alpha \beta}$.

Теорема 2 [14]. Для операторов $\mathscr{R}_{n}, n \in \mathbb{Z}_{+}$, равенство

$$
\operatorname{ad}_{\mathscr{R}_{n}, \mathscr{R}_{n-1}}^{1+1} f=0
$$

имеет место, если и только если коэффициенты оператора $\mathscr{R}_{n}$ и функиия $f$ удовлетворяют системе

$$
\begin{cases}\sum_{\alpha \leqslant \gamma,|\alpha| \leqslant K} r_{\alpha, \gamma-\alpha}^{n}=0, & |\gamma| \leqslant K, \\ \sum_{\alpha \leqslant \gamma,|\gamma|-K \leqslant|\alpha| \leqslant K} r_{\alpha, \gamma-\alpha}^{n}=0, & 1+K \leqslant|\gamma| \leqslant 2 K,\end{cases}
$$

$\left(\begin{array}{c}2 K+N \\ N\end{array}\right)$ нелинейных дифференииальных уравнений в частных производных. 
ДоКАЗАТЕЛЬСТВо. Вычислим

$$
\begin{aligned}
\operatorname{ad}_{\mathscr{R}_{n}, \mathscr{R}_{n-1} f} & =\sum_{\alpha,|\alpha| \leqslant K} u_{\alpha}^{n} \circ \partial^{\alpha} \circ f-f \circ \sum_{\alpha,|\alpha| \leqslant K} u_{\alpha}^{n-1} \circ \partial^{\alpha} \\
& =\sum_{\alpha,|\alpha| \leqslant K} u_{\alpha}^{n} \circ \sum_{\beta \leqslant \alpha}\left(\begin{array}{c}
\alpha \\
\beta
\end{array}\right)\left(\partial^{\alpha-\beta} f\right) \circ \partial^{\beta}-f \circ \sum_{\alpha,|\alpha| \leqslant K} u_{\alpha}^{n-1} \circ \partial^{\alpha} .
\end{aligned}
$$

Воспользовавшись формулой

$$
\sum_{\alpha,|\alpha| \leqslant K} \sum_{\beta \leqslant \alpha} u_{\alpha \beta}=\sum_{\alpha,|\alpha| \leqslant K} \sum_{\beta \geqslant \alpha,|\beta| \leqslant K} u_{\beta \alpha}
$$

получаем

$$
\operatorname{ad}_{\mathscr{R}_{n}, \mathscr{R}_{n-1}} f=\sum_{\alpha,|\alpha| \leqslant K}\left(\sum_{\beta \geqslant \alpha,|\beta| \leqslant K}\left(\begin{array}{l}
\beta \\
\alpha
\end{array}\right) u_{\beta}^{n} \circ\left(\partial^{\beta-\alpha} f\right)-f \circ u_{\alpha}^{n-1}\right) \circ \partial^{\alpha} .
$$

Положим

$$
g_{\alpha}^{n}=\sum_{\beta \geqslant \alpha,|\beta| \leqslant K}\left(\begin{array}{l}
\beta \\
\alpha
\end{array}\right) u_{\beta}^{n}\left(\partial^{\beta-\alpha} f\right)-u_{\alpha}^{n-1} f, \quad|\alpha| \leqslant K,
$$

тогда

$$
\operatorname{ad}_{\mathscr{R}_{n}, \mathscr{R}_{n-1}} f=\sum_{\alpha,|\alpha| \leqslant K} g_{\alpha}^{n} \circ \partial^{\alpha} .
$$

Отметим, что порядок оператора $\operatorname{ad}_{\mathscr{R}_{n}, \mathscr{R}_{n-1}} f$ не более чем $K-1$ в силу того, что при $|\alpha|=K$ имеет место равенство

$$
g_{\alpha}^{n}=u_{\alpha}^{n} f-u_{\alpha}^{n-1} f=\left(a_{\alpha}-a_{\alpha}\right) f=0 .
$$

Далее,

$$
\begin{aligned}
\operatorname{ad}_{\mathscr{R}_{n}, \mathscr{R}_{n-1}}^{2} f= & \operatorname{ad} \mathscr{R}_{n}, \mathscr{R}_{n-1}\left(\sum_{\alpha,|\alpha| \leqslant K} g_{\alpha}^{n} \circ \partial^{\alpha}\right)=\sum_{\alpha,|\alpha| \leqslant K} \operatorname{ad}_{\mathscr{R}_{n}, \mathscr{R}_{n-1}}\left(g_{\alpha}^{n} \circ \partial^{\alpha}\right) \\
= & \sum_{\alpha,|\alpha| \leqslant K}\left(\sum_{\beta,|\beta| \leqslant K} u_{\beta}^{n} \circ \partial^{\beta} \circ g_{\alpha}^{n} \circ \partial^{\alpha}-g_{\alpha}^{n} \circ \partial^{\alpha} \circ \sum_{\beta,|\beta| \leqslant K} u_{\beta}^{n-1} \circ \partial^{\beta}\right) \\
= & \sum_{\alpha,|\alpha| \leqslant K} \sum_{\beta,|\beta| \leqslant K}\left(u_{\beta}^{n} \circ \partial^{\beta} \circ g_{\alpha}^{n} \circ \partial^{\alpha}-g_{\alpha}^{n} \circ \partial^{\alpha} \circ u_{\beta}^{n-1} \circ \partial^{\beta}\right) \\
= & \sum_{\alpha,|\alpha| \leqslant K} \sum_{\beta,|\beta| \leqslant K}\left(u_{\alpha}^{n} \circ \partial^{\alpha} \circ g_{\beta}^{n} \circ \partial^{\beta}-g_{\alpha}^{n} \circ \partial^{\alpha} \circ u_{\beta}^{n-1} \circ \partial^{\beta}\right) \\
= & \sum_{\alpha,|\alpha| \leqslant K} \sum_{\beta,|\beta| \leqslant K}\left(u_{\alpha}^{n} \circ \sum_{\gamma \leqslant \alpha}\left(\begin{array}{l}
\alpha \\
\gamma
\end{array}\right)\left(\partial^{\alpha-\gamma} g_{\beta}^{n}\right) \circ \partial^{\gamma+\beta}\right. \\
& \left.-g_{\alpha}^{n} \circ \sum_{\gamma \leqslant \alpha}\left(\begin{array}{l}
\alpha \\
\gamma
\end{array}\right)\left(\partial^{\alpha-\gamma} u_{\beta}^{n-1}\right) \circ \partial^{\gamma+\beta}\right) \\
= & \sum_{\alpha,|\alpha| \leqslant K} \sum_{\beta,|\beta| \leqslant K} \sum_{\gamma \leqslant \alpha}\left(\begin{array}{l}
\alpha \\
\gamma
\end{array}\right)\left(u_{\alpha}^{n} \circ\left(\partial^{\alpha-\gamma} g_{\beta}^{n}\right)-g_{\alpha}^{n} \circ\left(\partial^{\alpha-\gamma} u_{\beta}^{n-1}\right)\right) \circ \partial^{\gamma+\beta} .
\end{aligned}
$$


Таким образом,

$$
\begin{aligned}
\operatorname{ad}_{\mathscr{R}_{n}, \mathscr{R}_{n-1}}^{2} f=\sum_{\alpha,|\alpha| \leqslant K} \sum_{\beta,|\beta| \leqslant K} \sum_{\gamma \geqslant \alpha,|\gamma| \leqslant K}\left(\begin{array}{l}
\gamma \\
\alpha
\end{array}\right)\left[u_{\gamma}^{n} \circ\left(\partial^{\gamma-\alpha} g_{\beta}^{n}\right)\right. & \\
& \left.-g_{\gamma}^{n} \circ\left(\partial^{\gamma-\alpha} u_{\beta}^{n-1}\right)\right] \circ \partial^{\alpha+\beta} .
\end{aligned}
$$

Для $\beta$ и $\alpha$ таких, что $|\beta| \leqslant K,|\alpha| \leqslant K$, положим

$$
r_{\alpha \beta}^{n}=\sum_{\gamma \geqslant \alpha,|\gamma| \leqslant K}\left(\begin{array}{l}
\gamma \\
\alpha
\end{array}\right)\left[u_{\gamma}^{n}\left(\partial^{\gamma-\alpha} g_{\beta}^{n}\right)-g_{\gamma}^{n}\left(\partial^{\gamma-\alpha} u_{\beta}^{n-1}\right)\right]
$$

тогда

$$
\operatorname{ad}_{\mathscr{R}_{n}, \mathscr{R}_{n-1}}^{2} f=\sum_{\alpha,|\alpha| \leqslant K} \sum_{\beta,|\beta| \leqslant K} r_{\alpha \beta}^{n} \circ \partial^{\alpha+\beta} .
$$

Далее, положив для мультииндекса $\gamma,|\gamma| \leqslant 2 K$,

$$
R_{\gamma}^{n}=\sum_{\substack{\alpha, \beta|\alpha+\beta=\gamma\\| \alpha|\leqslant K,| \beta \mid \leqslant K}} r_{\alpha \beta}^{n}
$$

имеем

$$
\operatorname{ad}_{\mathscr{R}_{n}, \mathscr{R}_{n-1}}^{2} f=\sum_{\gamma,|\gamma| \leqslant 2 K} R_{\gamma}^{n} \circ \partial^{\gamma}
$$

Заметим теперь, что

$$
R_{\gamma}^{n}= \begin{cases}\sum_{\alpha \leqslant \gamma,|\alpha| \leqslant K} r_{\alpha, \gamma-\alpha}^{n}, & |\gamma| \leqslant K, \\ \sum_{\alpha \leqslant \gamma,|\gamma|-K \leqslant|\alpha| \leqslant K} r_{\alpha, \gamma-\alpha}^{n}, & 1+K \leqslant|\gamma| \leqslant 2 K,\end{cases}
$$

и

$$
\operatorname{ad}_{\mathscr{R}_{n}, \mathscr{R}_{n-1}}^{1+1} f=0 \Longleftrightarrow R_{\gamma}^{n}=0, \quad 0 \leqslant|\gamma| \leqslant 2 K .
$$

При фиксированном $|\gamma|$ количество элементов $R_{\gamma}^{n}$ равно

$$
\left(\begin{array}{c}
|\gamma|+N-1 \\
N-1
\end{array}\right)
$$

Таким образом, число уравнений в системе равно

$$
\sum_{|\gamma|=0}^{2 K}\left(\begin{array}{c}
|\gamma|+N-1 \\
N-1
\end{array}\right)=\left(\begin{array}{c}
2 K+N \\
N
\end{array}\right)
$$

Теорема доказана.

УТВЕРЖДЕНИЕ 5. Для мультииндекса $\gamma$ такого, что $|\gamma|=2 K$ или $|\gamma|=2 K-1$, имеет место тождество $R_{\gamma}^{n}=0$. 
ДоКАЗАТЕЛЬСТВО. Пусть $|\gamma|=2 K$. Тогда

$$
R_{\gamma}^{n}=\sum_{\substack{\alpha, \beta|\alpha+\beta=\gamma\\| \alpha|=K,| \beta \mid=K}} r_{\alpha \beta}^{n}=0
$$

по свойству 1.

Если $|\gamma|=2 K-1$, то

$$
R_{\gamma}^{n}=\sum_{\substack{\alpha, \beta|\alpha+\beta=\gamma\\| \alpha|=K,| \beta \mid=K-1}} r_{\alpha \beta}^{n}+\sum_{\substack{\alpha, \beta|\alpha+\beta=\gamma\\| \alpha|=K-1,| \beta \mid=K}} r_{\alpha \beta}^{n}=\sum_{\substack{\alpha, \beta|\alpha+\beta=\gamma\\| \alpha|=K,| \beta \mid=K-1}}\left(r_{\alpha \beta}^{n}+r_{\beta \alpha}^{n}\right)=0
$$

по свойству 2. Доказательство завершено.

Отметим, что в теории изогюйгенсовых деформаций дифференциальных операторов [1], [2], [6], [15] однородньй гюйгенсов оператор обычно деформируется членами, имеюшими порядок меньше на два, чем у исходного оператора. В этой связи приводится следующее утверждение.

УТВЕРЖДЕНИЕ 6. Если в деформации $\mathscr{R}_{n}$ коэффициенты $u_{\alpha}^{n},|\alpha|=K-1$, нулевые, то из условия 1-калибровочной эквивалентности операторов $\mathscr{R}_{n}$ u $\mathscr{R}_{n-1}$

$$
\operatorname{ad}_{\mathscr{R}_{n}, \mathscr{R}_{n-1}}^{1+1} f=0
$$

следует, что функиия $f$ удовлетворяет системе $\left(\begin{array}{c}2 K+N-3 \\ N-1\end{array}\right)$ дифференииальных уравнений

$$
\sum_{\substack{\alpha, \beta|\alpha+\beta=\gamma\\| \alpha|=| \beta \mid=K-1}} \sum_{i, j=1}^{N}\left(\alpha_{i}+1\right)\left(\beta_{j}+1\right) a_{\alpha+\overline{1}_{i}} a_{\beta+\overline{1}_{j}} \frac{\partial^{2} f}{\partial x_{i} \partial x_{j}}=0,
$$

әде мультииндекс $\overline{1}_{i}$ состоит из нулевых әлементов за исключением единииы на $i$-й позичии.

ДокАЗАТЕЛЬСТво. Для мультииндексов $\gamma$ таких, что $|\gamma|=2 K-2$, имеем

$$
\begin{aligned}
R_{\gamma}^{n} & =\sum_{\substack{\alpha, \beta|\alpha+\beta=\gamma\\
| \alpha|=K,| \beta \mid=K-2}} r_{\alpha \beta}^{n}+\sum_{\substack{\alpha, \beta|\alpha+\beta=\gamma\\
| \alpha|=K-1,| \beta \mid=K-1}} r_{\alpha \beta}^{n}+\sum_{\substack{\alpha, \beta|\alpha+\beta=\gamma\\
| \alpha|=K-2, \beta|=K}} r_{\alpha \beta}^{n} \sum_{\substack{\alpha, \beta|\alpha+\beta=\gamma\\
| \alpha|=K,| \beta \mid=K-2}}\left(r_{\alpha \beta}^{n}+r_{\beta \alpha}^{n}\right)+\sum_{\substack{\alpha, \beta|\alpha+\beta=\gamma\\
| \alpha|=K-1,| \beta \mid=K-1}} r_{\alpha \beta}^{n}=\sum_{\substack{\alpha, \beta|\alpha+\beta=\gamma\\
| \alpha|=K-1,| \beta \mid=K-1}}^{n} r_{\alpha \beta}^{n} .
\end{aligned}
$$

Остается выяснить явный вид элементов $r_{\alpha \beta}^{n}$ при $|\alpha|=K-1,|\beta|=K-1$. Из определения имеем

$$
\begin{aligned}
r_{\alpha \beta}^{n} & =u_{\alpha}^{n} g_{\beta}^{n}-g_{\alpha}^{n} u_{\beta}^{n-1}+\sum_{\gamma>\alpha,|\gamma|=K}\left(\begin{array}{c}
\gamma \\
\alpha
\end{array}\right)\left[u_{\gamma}^{n}\left(\partial^{\gamma-\alpha} g_{\beta}^{n}\right)-g_{\gamma}^{n}\left(\partial^{\gamma-\alpha} u_{\beta}^{n-1}\right)\right] \\
& =\sum_{\gamma>\alpha,|\gamma|=K}\left(\begin{array}{c}
\gamma \\
\alpha
\end{array}\right) u_{\gamma}^{n}\left(\partial^{\gamma-\alpha} g_{\beta}^{n}\right)=\sum_{i=1}^{N}\left(\begin{array}{c}
\alpha+\overline{1}_{i} \\
\alpha
\end{array}\right) u_{\alpha+\overline{1}_{i}}^{n}\left(\partial^{\overline{1}_{i}} g_{\beta}^{n}\right) \\
& =\sum_{i=1}^{N}\left(\alpha_{i}+1\right) a_{\alpha+\overline{1}_{i}}\left(\frac{\partial}{\partial x_{i}} g_{\beta}^{n}\right) .
\end{aligned}
$$


Далее, для $\beta,|\beta|=K-1$, имеем

$$
\begin{aligned}
g_{\beta}^{n} & =u_{\beta}^{n} f-u_{\beta}^{n-1} f+\sum_{\gamma>\beta,|\gamma|=K}\left(\begin{array}{l}
\gamma \\
\beta
\end{array}\right) u_{\gamma}^{n}\left(\partial^{\gamma-\beta} f\right) \\
& =\sum_{j=1}^{N}\left(\begin{array}{c}
\beta+\overline{1}_{j} \\
\beta
\end{array}\right) u_{\beta+\overline{1}_{j}}^{n}\left(\partial^{\overline{1}_{j}} f\right)=\sum_{j=1}^{N}\left(\beta_{j}+1\right) a_{\beta+\overline{1}_{j}}\left(\frac{\partial f}{\partial x_{j}}\right) .
\end{aligned}
$$

Тогда при $\alpha, \beta,|\alpha|=|\beta|=K-1$, имеем

$$
r_{\alpha \beta}^{n}=\sum_{i, j=1}^{N}\left(\alpha_{i}+1\right)\left(\beta_{j}+1\right) a_{\alpha+\overline{1}_{i}} a_{\beta+\overline{1}_{j}} \frac{\partial^{2} f}{\partial x_{i} \partial x_{j}} .
$$

Доказательство завершено.

ЗАмечАниЕ. В случае $N=1, x \in \mathbb{R}$ система уравнений утверждения 6 принимает наиболее простой вид

$$
\frac{d^{2} f}{d x^{2}}=0
$$

4. Примеры. В этом пункте приводятся некоторые примеры калибровочно эквивалентных операторов.

ПримеР 1 (специальный дифференциальньй многочлен, [14]). Пусть $\mathscr{R}_{0}$ - однородный дифференциальный оператор порядка $K$ с постоянными коэффициентами на пространстве $\mathbb{R}^{N}=\left\{(y, x) \mid y \in \mathbb{R}, x \in \mathbb{R}^{N-1}\right\}$. Представим его в виде

$$
\mathscr{R}_{0}=\sum_{k=0}^{K} \mathscr{A}_{k} \circ \frac{\partial^{k}}{\partial y^{k}}
$$

где $\mathscr{A}_{k}$ - линейные дифференциальные операторы на $\mathbb{R}^{N-1}=\{x\}$ порядка $K-k \mathrm{c}$ постоянньми коэффициентами. Тогда для операторов

$$
\mathscr{R}_{n}=\mathscr{R}_{0}+\sum_{k=0}^{K-2}\left(\sum_{p=2}^{K-k}(-1)^{p-1}(p-1)\left(\begin{array}{c}
p+k \\
k
\end{array}\right) \frac{(n, p)}{y^{p}} \circ \mathscr{A}_{p+k}\right) \circ \frac{\partial^{k}}{\partial y^{k}}, \quad n \in \mathbb{Z}_{+},
$$

где $(n, p)=n(n+1) \cdots(n+p-1)-$ символ Похгаммера, вьполняются условия теоремы 1 c $f(y, x)=y$.

Таким образом, оказывается, что оператор $\mathscr{R}_{n} n$-калибровочно эквивалентен специальному дифференциальному многочлену $\mathscr{R}_{0}$ :

$$
\operatorname{ad}_{\mathscr{R}_{n}, \mathscr{R}_{0}}^{n+1} f^{n}(y, x)=0, \quad f^{n}(y, x)=y^{n} .
$$

При этом коэффициенты, используемые в деформации специального дифференциального многочлена, обобшают потенциалы Штелльмахера [3].

В случае $K=2$ указанные в примере деформации (и более общие) использовались в работах [15], [16] для осуществления изогюйгенсовых деформаций линейных дифференциальных операторов в частных производных высоких порядков. 
ПримеР 2 (оператор Лапласа на плоскости). Этот пример приводится для иллюстрации совместного использования теорем $1^{\prime}$ и 2 . Окончательньй результат, полученный другими средствами, содержится в [1], [2], [5], [6].

Пусть

$$
\Delta=\frac{\partial^{2}}{\partial x_{1}^{2}}+\frac{\partial^{2}}{\partial x_{2}^{2}} \equiv \mathscr{R}_{0,0}
$$

- оператор Лапласа на плоскости $\mathbb{R}^{2}$.

Рассмотрим деформацию оператора Лапласа

$$
\mathscr{R}_{n, m}=\Delta+\mathscr{U}_{n, m}\left(x_{1}, x_{2}\right)
$$

при помощи потенциала, зависящего от двух переменных и двух параметров $n, m \in \mathbb{Z}_{+}$, с начальньп условием

$$
\mathscr{U}_{0,0}\left(x_{1}, x_{2}\right)=0
$$

Согласно теореме $1^{\prime}$, потребовав выполнения равенства

$$
\operatorname{ad}_{\mathscr{R}_{0, m}, \mathscr{R}_{0, m-1}}^{1+1} f_{2}=0
$$

получим

$$
\mathscr{U}_{0, m}\left(x_{1}, x_{2}\right)=-\frac{m(m+1)\left(a_{2}^{2}+b_{2}^{2}\right)}{\left(a_{2} x_{1}+b_{2} x_{2}\right)^{2}} .
$$

Теперь потребуем выполнение равенства

$$
\operatorname{ad}_{\mathscr{R}_{n, m}, \mathscr{R}_{n-1, m}}^{1+1} f_{1}=0
$$

с начальньм условием, задаваемым $\mathscr{U}_{0, m}\left(x_{1}, x_{2}\right)$.

В результате получаем потенциал $\mathscr{U}_{n, m}\left(x_{1}, x_{2}\right)$ и соответствующую изодеформацию

$$
\mathscr{R}_{n, m}=\frac{\partial^{2}}{\partial x_{1}^{2}}+\frac{\partial^{2}}{\partial x_{1}^{2}}-\frac{n(n+1)}{x_{1}^{2}}-\frac{m(m+1)}{x_{2}^{2}}
$$

с точностью до элементарных преобразований, сохраняющих вид оператора Лапласа на плоскости. При этом имеет место следующая $(n+m)$-калибровочная эквивалентность:

$$
\operatorname{ad}_{\mathscr{R}_{n, m}, \Delta}^{n+m+1}\left(f_{1}^{n} f_{2}^{m}\right)=0, \quad n, m \in \mathbb{Z}_{+}, \quad f_{1}=x_{1}, \quad f_{2}=x_{2} .
$$

Как известно, потенциалы

$$
\mathscr{U}_{n, m}\left(x_{1}, x_{2}\right)=-\frac{n(n+1)}{x_{1}^{2}}-\frac{m(m+1)}{x_{2}^{2}}
$$

суть в точности потенциалы Лагнезе-Штелльмахера [4] для оператора Лапласа на плоскости. Добавлениеэтих потенциалов к волновому оператору на пространствах большой пространственной размерности позволяет получить некоторые изогюйгенсовы деформации волнового оператора [4], [1]. 


\section{СПИСОК ЦИТИРОВАННОЙ ЛИТЕРАТУРЫ}

[1] Берест Ю. Ю., Веселов А. П. Принцип Гюйгенса и интегрируемость // УМН. 1994. T. 49. № 6. C. 8-78.

[2] Berest Y. The problem of lacunas and analysis on root systems // Trans. Amer. Math. Soc. 2000. V. 352. № 8. P. 3743-3776.

[3] Stellmacher K. L. Ein Beispeil einer Huygennchen Differentialgleichung // Nachr. Akad. Wiss. Göttingen Math. -Phys. Kl. 1953. V. 10. P. 133-138.

[4] Lagnese J. E., Stellmacher K. L. A method of generating classes of Huygens' operators // J. Math. Mech. 1967. V. 17. № 5. P. 461-472.

[5] Berest Y., Molchanov Y. Fundamental solution for partial differential equations with reflection group invariance // J. Math. Phys. 1995. V. 36. № 8. P. 4324-4339.

[6] Берест Ю. Ю., Веселов А. П. Проблема Адамара и группы Кокстера: новые примеры гюйгенсовых уравнений // Функцион. анализ и его прилож. 1994. V. 28. №1. Р. 3-15.

[7] Berest Y. Hierarchies of Huygens' operators and Hadamard's conjecture // Acta Appl. Math. 1998. V. 53. P. 125-185.

[8] Бабич В. М. Анзатц Адамара, его аналоги, обобщения, приложения // Алгебра и анализ. 1991. T. 3. № 5. C. 1-37.

[9] Wilson G. Bispectral commutative ordinary differential operators // J. Reine Angew. Math. 1993. V. 442. P. 177-204.

[10] Berest Y. Y., Loutsenko I. M. Huygens' principle in Minkowski spaces and soliton solutions of the Korteweg-de Vries equation // Comm. Math. Phys. 1997. V. 190. P. 113-132.

[11] Berest Y. Solution of a restricted Hadamard problem on Minkowski spaces // Comm. Pure. Appl. Math. 1997. V. 50. P. 1019-1052.

[12] Khekalo S. The gauge relation of differential operators and Huygens' principle // Day on Diffraction. Saint-Petersburg, 2002. P. 32-34.

[13] Хэкало С.П. Калибровочная эквивалентность дифференциальных операторов в частньх производных // Международная конференция по дифференциальным уравнениям и динамическим системам. Тезисы докл. Суздаль, 2002. С. 138-140.

[14] Khekalo S. The gauge related differential operators // Day on Diffraction. Saint-Petersburg, 2003. P. 42-43.

[15] Хэкало С. П. Изогюйгенсовы деформации однородных дифференциальных операторов, связанных со специальным конусом ранга три // Матем. заметки. 2001. Т. 70. №6. С. 927-940.

[16] Хэкало С. П. Потенциалы Рисса в пространстве прямоугольных матриц и изогюйгенсова деформация оператора Кэли-Лапласа // Докл. РАН. 2001. Т. 376. № 2. С. 168-170.

Санкт-Петербургское отделение

Математического института им. В.А. Стеклова РАН

E-mail: khekalo@pdmi.ras.ru

Поступило

21.01.2004

Исправленный вариант

17.11.2004 